\title{
THE THERMAL ACCOMMODATION COEFFICIENT OF GRAPHITE
}

\author{
KENRICK L. DAY \\ Astronomy Dept., The Ohio State University, Columbus, Ohio 43210, U.S.A.*
}

\begin{abstract}
An experiment has been performed to determine the thermal accommodation coefficients for the gases hydrogen, oxygen, methane, and carbon dioxide on graphite, in the temperature range 273-77 K. The experimental results are compared to those indirectly predicted in a theoretical paper by Hollenbach and Salpeter, and agreement is found to be satisfactory. A sticking coefficient for atomic hydrogen on a graphite grain is derived which would support the conclusion that recombination of hydrogen on grain surfaces could be a significant process. The very high measured thermal accommodation coefficients for the heavier gases supports the popular assumption that retention of such gases on grain surfaces should be considered extremely likely.
\end{abstract}

\section{Introduction}

The thermal accommodation coefficient (hereafter abbreviated TAC) is a measure of the efficiency of energy transfer between a gas and a solid. It is of interest in discussing the condensation of various gases on interstellar grains, which in this paper are assumed to be graphite. The TAC has often been confused with the sticking coefficient, which measures the actual fraction of atoms condensing, while the TAC gives the fractional energy transfer. Both these quantities tend to rise with decreasing temperature, with the TAC generally higher than the sticking coefficient. For a cold surface, or one on which the surface adsorptive potential is greater than the thermal energy, the main variable influencing the TACs and sticking coefficients is the energy of the incoming gas particle.

\section{Experimental Details}

The experiment was performed via the hot-wire, low pressure method. For a comprehensive discussion, the reader is referred to Wachmann (1962) and Thomas (1967). Basically, a tube of cylindrical symmetry is constructed with the substrate material in the form of a fine filament running axially. A graphite filament was secured from the Poco Graphite Company of Decatur, Texas. This was a polycrystalline material graphitized from coke at $2500^{\circ} \mathrm{C}$. The manufacturers estimate that at this temperature of graphitization, approximately half the coke may be considered to have been converted to graphite. Impurities present amount to about $200 \mathrm{ppm}$, the primary ones being $\mathrm{Fe}, \mathrm{Si}$, and $\mathrm{V}$. The material may be regarded as nearly perfectly isotropic, with grain sizes under one nil. $L_{c}$ is given to be $340 \AA$, and the BET area is approximately $0.336 \mathrm{~m}^{2} \mathrm{~g}^{-1}$.

The basic procedure is to heat the filament electrically in a vacuum by passing a

* Current address: Physics Dept., University of Arizona, Tucson, Ariz. 85721, U.S.A.

Greenberg and Van de Hulst (eds.), Interstellar Dust and Related Topics, 311-315, All Rights Reserved. Copyright (C) 1973 by the IAU. 
small current through it, and measure the power loss in the filament. A small amount of sample gas at a measured pressure $(0.01$ to 0.05 torr) is then introduced and the filament is returned to its previous temperature. This will require more power than before due to heat loss to the gas. By subtracting the vacuum power loss, one finds the net power loss to the gas; this measured quantity, when compared to a theoretical maximum calculated from kinetic theory, gives the TAC.

A few experimental considerations must be mentioned. It is necessary that the filament be of fine diameter so that it does not appreciably disturb the temperature distribution of the gas particles impinging on it; this allows one to assume that all particles striking the filament are in thermal equilibrium with the walls of the experimental tube, which is placed in a constant temperature bath. The filament diameter in this case was just under 9 mils. A filament would tend to lose heat into its end supports. The amount of this loss is unfortunately not the same in vacuo and in the sample gas, because the gas changes the heat distribution along it. The reader is referred to Thomas and Olmer (1943). This was avoided by placing small auxiliary heaters at either end of the tube, which held the end supports at a constant temperature equal to the filament's. Thus there was no heat lost to the ends either in vacuo or in the sample gas. This temperature was monitored by means of iron-constantan thermocouples.

The temperature of the filament itself was found by measuring its resistance at any given moment and then comparing this to a large scale graph of temperature vs resistance which was previously prepared for this sample. The resistance was found by measuring the voltage drop across the filament and across a standard resistor in series with the filament and then applying Ohm's law. For most experimental runs the graphite was maintained at ten degrees Centigrade above it surroundings.

Considerable inconsistancy in TAC measurements has been noted in the past, and much of it has apparently been due to variations in surface cleanliness. Current cleaning techniques include flashing the filament or the use of various getters. Because of several problems, this was not possible with the sample used in this experiment, so that the surface must be regarded as having an adsorbed layer of sample gas on it during measurement. A molecular sieve sorption pump was used to minimize external contamination. It was also noted that no progressive alteration on the surface was taking place as the measured TAC values were repeatable over a period of several months.

\section{Results of the Experiment}

For $\mathrm{H}_{2}$ on graphite, the following average TACs were found. At a gas temperature of $273 \mathrm{~K}, \mathrm{TAC}=0.36$. At $195 \mathrm{~K}, \mathrm{TAC}=0.38$. At $77 \mathrm{~K}, \mathrm{TAC}=0.82$.

Results for oxygen, methane, and carbon dioxide were obtained only at the temperature of water ice, or about $273 \mathrm{~K}$. The following results were noted. Oxygen, $\mathrm{TAC}=0.96$. Methane, $\mathrm{TAC}=0.78$. Carbon dioxide, $\mathrm{TAC}=0.90$. For all the above, the p.e. is \pm 0.009 . 


\section{Interpretation of Experimental Data and Conclusions}

It is informative to campare the measured TACs for $\mathrm{H}_{2}$ on graphite to theoretical predictions by Hollenbach and Salpeter (1970). These authors did not actually make predictions for the hydrogen/graphite combination, but developed an analytic formula which should fit many situations in which the energy of the substrate material is small compared to the surface adsorption potential. This should be the case in an interstellar cloud and for the temperatures used for this paper in the laboratory. For this circumstance, the variable of note is the energy of the incoming gas particle.

In order to make a prediction it is necessary to know the adsorption potential for $\mathbf{H}_{2}$ on graphite. This is of critical importance to the problem, and existing data is badly in need of confirmation. The value adopted is that calculated by Ross and Oliver (1964) for carbon black graphitized at $2700 \mathrm{~K}$, which is $960 \mathrm{cal} \mathrm{mole}^{-1}$. The results of the experiment and the curve calculated from the work of Hollenbach and Salpeter (1970) are shown in Figure 1. It is noted that the fit is fairly reasonable, being just a little too shallow.

Hollenbach and Salpeter (1970) produce formulae for both sticking and thermal accomodation coefficients in terms of a single parameter which they call gamma. If one accepts the fit of the theoretical curve to the TAC data, it is an indication that gamma is not far from being correct.

To plot a curve for the sticking coefficient of hydrogen vs temperature, gamma must be modified somewhat. One would generally be interested in the case of impinging atomic, rather than molecular, hydrogen. However, the adsorptive potential for atomic hydrogen on graphite has never been measured. According to Augason (1970), we may assume that the ratio of the adsorption potentials should be the same

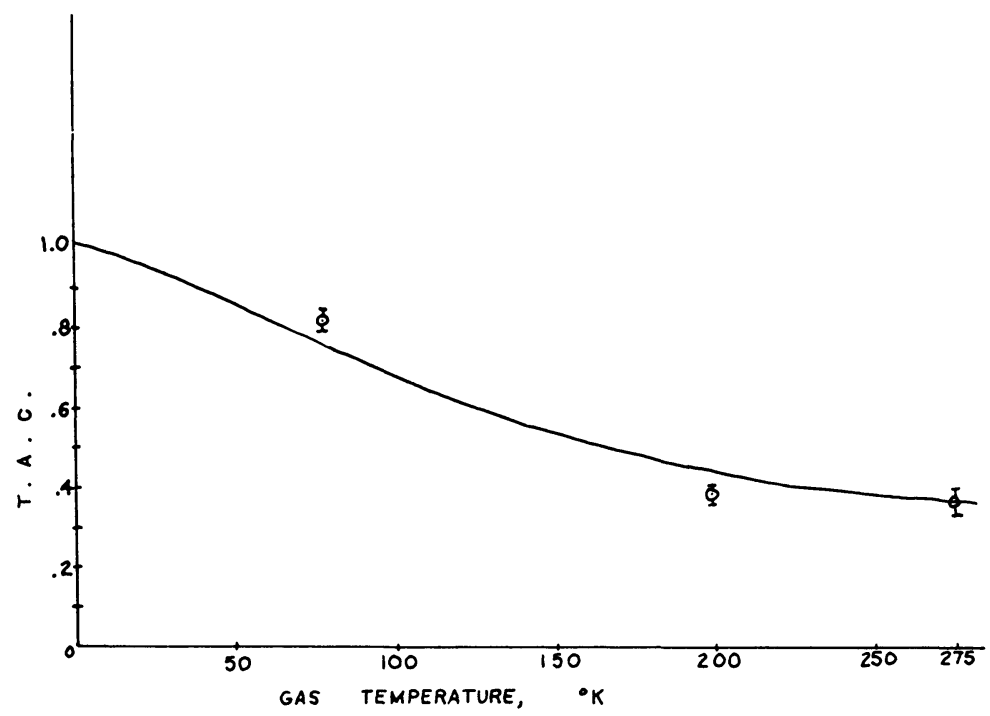

Fig. 1. TAC vs temperature for $\mathrm{H}_{2}$. - Theory. Experimental values. 


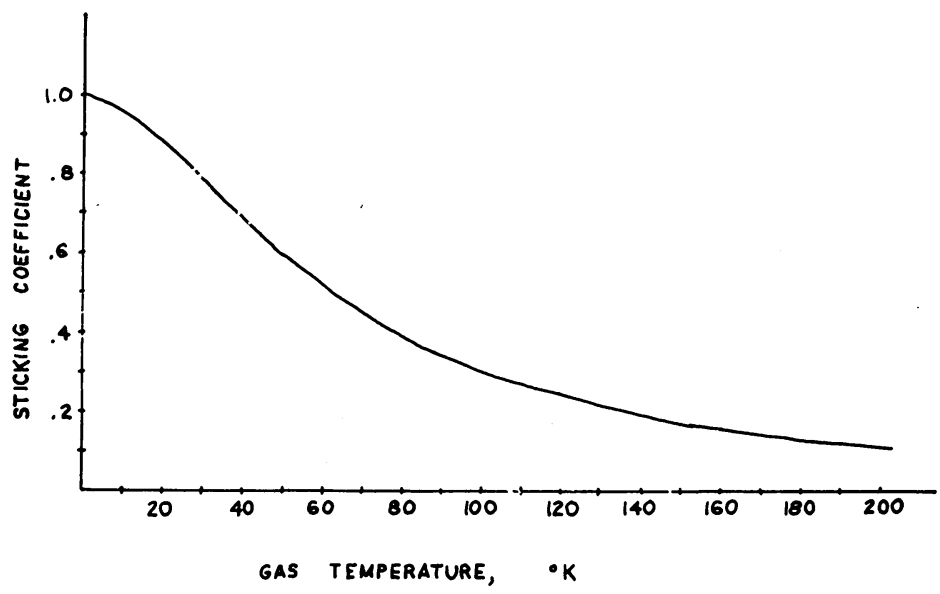

Fig. 2. Computed sticking coefficient for atomic hydrogen on a graphite grain.

as the ratio of the polarizabilities, 0.84 , so the adsorption potential of $\mathrm{H}$ on graphite should be 810 cal mole $^{-1}$.

Using this figure for the adsorption potential, the curve appearing in Figure 2 has been generated, which should represent the sticking probability of atomic hydrogen on graphite. The sticking coefficient is of use in determining under which conditions one might expect to find production of $\mathrm{H}_{2}$ by recombination on grain surfaces. A basic requirement is that new hydrogen atoms stick to the surface at a rate equal to or greater than the departure rate. This may be used to derive a maximum grain temperature for which the condition holds. According to Augason (1970), this is:

$$
\left(T_{\text {grain }}\right)_{\max }=\frac{U}{R}\left(\ln \frac{v_{0}}{a n_{\mathrm{H}} v S}\right)^{-1},
$$

where $U$ is the adsorptive potential, $R$ is the gas constant, $v_{0}$ is the characteristic vibration frequency, $a$ is the sticking coefficient, $n_{\mathrm{H}}$ is the number density of $\mathrm{H}$ atoms, $v$ is the average velocity of $\mathrm{H}$ atoms, and $S$ is grain surface area.

According to Ross and Oliver (1964) the characteristic vibration frequency for $\mathrm{H}$ on graphite is $10^{13} \mathrm{~s}^{-1}$. For a gas temperature of $100 \mathrm{~K}$, the average velocity is $1.45 \times 10^{5}$ $\mathrm{cm} \mathrm{s}^{-1}$. Further, it will be assumed that the number density is $10 \mathrm{~cm}^{-3}$ and that the grain surface area is $10^{-8} \mathrm{~cm}^{2}$. The sticking coefficient derived in this work for an incident particle temperature of $100 \mathrm{~K}$ is 0.30 . This yields a maximum allowable grain temperature of $11.53 \mathrm{~K}$.

The question of grain temperatures has been debated for some time, but in the opinion of the author the work of Field (1969) is the most comprehensive to date. Taking into account all known cooling mechanisms, he derives minimum possible temperatures for graphite grains of from 8.9 to $9.3 \mathrm{~K}$. This would appear to leave a significant range of temperatures in which molecular hydrogen could form on graphite grains. 
The experimental data for the gases oxygen, methane, and carbon dioxide tend to confirm what people have been assuming at least since Van de Hulst (1949). This is that the sticking coefficients for these gases should be unity. The measured TACs for these gases at $273 \mathrm{~K}$ range from 0.78 to 0.96 . While the sticking coefficients would probably be lower by a factor of about a third at this temperature, both coefficients rise with decreasing temperature so that by $100 \mathrm{~K}$ a coefficient of nearly unity should prevail. It would appear that the conclusions of Williams (1968) are correct; molecule accretion by graphite grains may actually begin before the grain is completely free of the atmosphere of the parent star, and nearly all impinging molecules should stick.

\section{Acknowledgements}

The author wishes to acknowledge helpful letters from Drs B. Donn and G. C. Augason, the support of Dr T. P. Roark and Mr Roy S. Tucker of the Ohio State University, and partial funding by NSF grant GP-7616.

\section{References}

Augason, G. C.: 1970, Astrophys. J. 162, 463.

Field, G. B.: 1969, Monthly Notices Roy. Astron. Soc. 144, 411.

Hollenbach, D. and Salpater, E. E.: 1970, J. Chem. Phys. 53, 79.

Ross, S. and Oliver, J. P.: 1964, On Physical Adsorption, Interscience Publishers, New York, p. 236.

Thomas, L. B.: 1967, in Saltsburg et al. (eds.), Fundamentals of Gas-Surface Reactions, Academic Press, New York, p. 346.

Thomas, L. B. and Olmer, F. G.: 1943, J. Am. Chem. Soc. 65, 1036.

Van de Hulst, H. C.: 1949, Recherches Astronomiques de l'Observatoire d'Urecht XI, Part 2, 1.

Wachman, H. Y.: 1962, J. Am. Rocket Soc. 32, 2.

Williams, D. A.: 1968, Astrophys. J. 151, 935. 